\title{
Photoconductive zinc oxide-composite paper by pilot paper machine manufacturing
}

Mats Sandberg, Daniel Tordera, Hjalmar Granberg, Anurak Sawatdee, Dina Dedic, Magnus Berggren and Magnus P J onsson

The self-archived postprint version of this journal article is available at Linköping University Institutional Repository (DiVA):

http:/ / urn.kb.se/ resolve?urn=urn:nbn:se:liu:diva- 143683

N.B.: When citing this work, cite the original publication.

Sandberg, M., Tordera, D., Granberg, H., Sawatdee, A., Dedic, D., Berggren, M., J onsson, M. P, (2016), Photoconductive zinc oxide-composite paper by pilot paper machine manufacturing, Flexible and printed electronics, 1(4), . https:// doi.org/ 10.1088/2058-8585/ 1/4/044003

Original publication available at:

https:/ / doi.org/ 10.1088/2058-8585/1/4/044003

Copyright: IOP Publishing (Hybrid Open Access)

http:// www.iop.org/ 


\title{
Photoconductive Zinc Oxide-Composite Paper by Pilot Paper Machine Manufacturing
}

\author{
Mats Sandberg ${ }^{\S, 1,2}$, Daniel Tordera ${ }^{\S, 1}$, Hjalmar Granberg ${ }^{3}$, Anurak Savatdee $^{2}$, Dina Dedic ${ }^{3}$, \\ Magnus Berggren ${ }^{1}$ and Magnus P. Jonsson ${ }^{\star 1}$ \\ ${ }^{1}$ Laboratory of Organic Electronics, ITN, Linköping University, SE-601 74 Norrköping, Sweden \\ 2 Acreo Swedish ICT, Box 787, SE-601 17 Norrköping, Sweden \\ 3 Innventia AB, Box 5604, SE-114 28 Stockholm, Sweden \\ $\S$ These authors contributed equally to this work. \\ * Corresponding author email: magnus.jonsson@liu.se
}

\begin{abstract}
Smart materials can be used for a wide variety of applications, including sensing and energy harvesting. Implementation of smart materials in large area devices requires scalable manufacturing. The use of paper-making techniques would offer an enormous production capacity, allowing for low-cost and large-scale manufacturing. In this work we present successful pilot scale paper machine manufacturing of functional composite papers $(100 \mathrm{~m} / \mathrm{min}$ with a web width of $30 \mathrm{~cm}$ ) based on cellulose fibres and commercial tetrapodal zinc oxide microwhiskers (ZnO-Ts). Carbon electrodes could successfully be printed on the paper, to form complete electronic devices where the paper itself is the active material. This enabled development of a ZnO-composite paper photosensor, where we characterized its stability, sensitivity and speed. The devices show excellent photosensing properties over a wide range of light irradiances (0.011 Sun), including short response times ( $10 \mathrm{~s})$ and long-term stability. Under simulated sunlight and a bias voltage of $1 \mathrm{~V}$, small $\left(0.5 \mathrm{~cm}^{2}\right)$ two-probe interdigitated photosensor devices provided $12 \mu \mathrm{A}$ photocurrent. Under the same conditions, four-probe measurements of the composite paper show a sheet resistance of $6.9 \cdot 10^{7} \Omega / \mathrm{sq}$. Four-probe measurements also demonstrates that the paper conductivity varies linearly with light irradiance. To the best of our knowledge, this is the first example of pilot paper machine production of an optoelectronic paper, demonstrating the potential for large-scale paper manufacturing of active smart paper from low-cost industrial bulk materials.
\end{abstract}

\section{Introduction}

Scalability in manufacturing and low cost are typical prerequisites for the successful implementation of (smart) materials in large area or large volume devices. The possibility to use paper manufacturing methods would be particularly attractive considering the huge production capacity of paper making machines (more than $100 \mathrm{~km} / \mathrm{h}$ ), the low cost of the starting material (around $0.1 \mathrm{cent} / \mathrm{dm}^{2}$ ) and its environmental-friendly nature [1]. All paper in the world together form the largest surface ever produced by mankind, with about 400 million metric tons produced by year (2013 data) [2].

The most common current approach to low-cost and large-area electronic devices is printed electronics. The principle takes advantage of low-cost material deposition using printing and coating techniques [3,4]. However, if substrates and printing inks are too expensive, this advantage is lost. Here, we explore an alternative route to low-cost large-area functional devices based on paper machine manufacturing. Optoelectronic papers are produced in a pilot paper 
machine and used as active substrates for manufacturing of optoelectronic devices by subsequent screen-printing of carbon electrodes and circuits onto the paper.

The active components in our papers are tetrapodal zinc oxide microstructures (ZnO-Ts). $\mathrm{ZnO}$ is an n-type semiconductor with a bandgap of $3.37 \mathrm{eV}$ and it is known for its photoconductive properties. For example, ZnO-based coatings on so-called electrofax papers for electrophotographic processes (xerography) were used for printing and reproduction until the 1980s [5,6]. The mechanism of photoconductivity in ZnO is related to photogeneration of electron-hole pairs and adsorption and (photo-)desorption of oxygen onto the material [7-9]. At dark conditions, oxygen molecules adsorbed on the material capture free electrons and create a low-conductive dark state. Upon illumination, holes from electron-pair generation react with and release the oxygen. This leaves non-paired free electrons and a corresponding high-conductive state. The material has a wide applicability and has been used in chemical sensors, as photosensors, detectors for reducing and oxidizing gases, in filters, as photocatalysts, and in various optoelectronic devices [10-15]. In addition, there is a strong interest in ZnO composites due to its high abundance, low cost and safety, since $\mathrm{ZnO}$ is a material generally recognized as safe (GRAS) [16]. Low-cost photosensing, and monitoring of UV exposure in particular, could be important in a many applications. For example, high UV-doses can be harmful to human skin, and is associated with health risks and cancer, while medium and low UV doses can benefit health $[17,18]$. UV-B radiation also affects plants and primary producers on aquatic ecosystems $[19,20]$. On the other hand, UV-light exposure can be used to preserve food [21,22] and sterilize water [23]. Availability of low-cost devices that can monitor UV-exposure doses could thus benefit health, crop production, reduce food waste and facilitate water purification.

Photosensors based on ZnO can be manufactured by a variety of deposition and patterning methods [7,24-26], even by simply drawing graphite electrodes by a pencil on top of a ZnO-based coatings [27] or on flexible materials such as cloth [28] or paper [29,30]. However, the ZnO-composite papers reported in the literature were not obtained using large-scale manufacturing, limiting their low-cost and applicability. Here, we present the first ZnO-composite paper made by large-scale paper machining and demonstrate its applicability for photosensing. We characterized the sensors using a light source that emulates solar illumination in order to evaluate the concept for low-cost monitoring of sun exposure. The composite paper shows high photoresponse in a wide range of light intensities (0.01 Sun to 1 Sun) with an excellent stability over time. Moreover, we chose to work with ZnO-Ts, which are manufactured on an industrial scale and available in bulk quantities at a low price ( $<100 \mathrm{USD} / \mathrm{kg}$ ), as opposed to more complex forms of nanostructured $\mathrm{ZnO}$ that have been used in photosensors and other applications [10].

\section{Materials and methods}

\subsection{Pilot Paper Machine manufacturing}

\section{Pilot Paper Machine}

The composite paper was manufactured on the FEX pilot paper machine at Innventia $A B$, in Stockholm, Sweden. A simplified schematic illustration of the manufacturing process is presented in figure 1(a), while figure 1(b) shows a photo of the FEX paper machine, as previously described in works by Roding et al. [31] and Nordström [32]. The FEX pilot paper machine is designed so that the machine sections and the material flow is similar to that of a fullscale paper machine. For example, it is possible to recirculate the white water back to the inlet flow. The paper machine was equipped with a hydraulic headbox from Andritz, shown in figure 1(c). To make experiments possible with a suitable quantity of raw materials, the web width of the FEX machine is $30 \mathrm{~cm}$, compared with full-scale paper machines where the web typically is 
several meters wide. To avoid potential contamination of ZnO-Ts particles, the drying section was disconnected and paper samples were rolled up on bobbins, shown in Figure 1(d). These bobbins were then transferred to a mobile drying section placed in a compartment confining dust emissions. After drying, the paper roll was cut to approximately one-meter-long sheets. In this experiment, the paper machine was running at a web speed of $100 \mathrm{~m} / \mathrm{min}$. Since this is still slow for paper-making, there is potential for further up-scaling. As described in detail below, the pulp composition was changed during production to produce seven paper compositions. Since the white water is re-circulated, paper samples were collected 20 minutes after the composition of the feed was changed. For each sample, approximately 60 meter of paper was collected.

a)

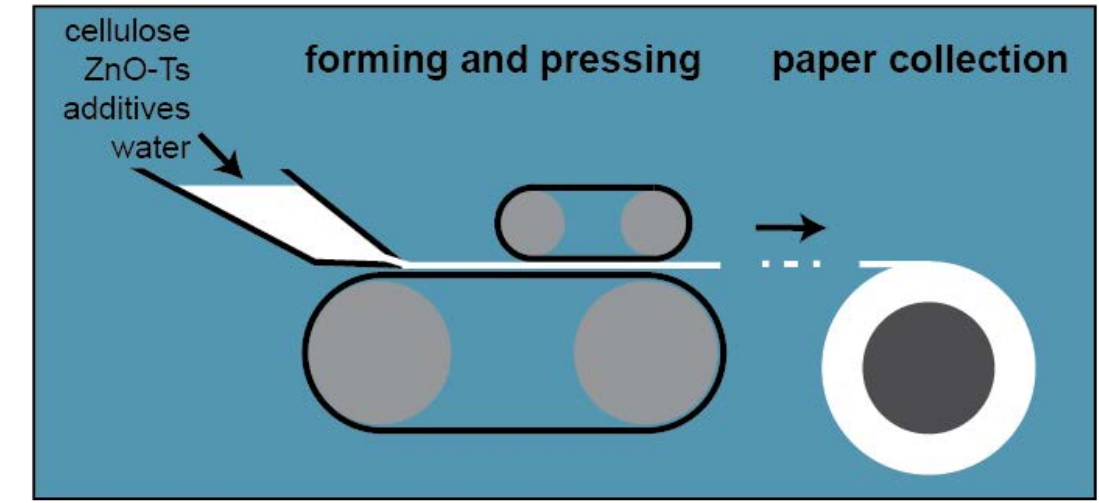

b)

c)
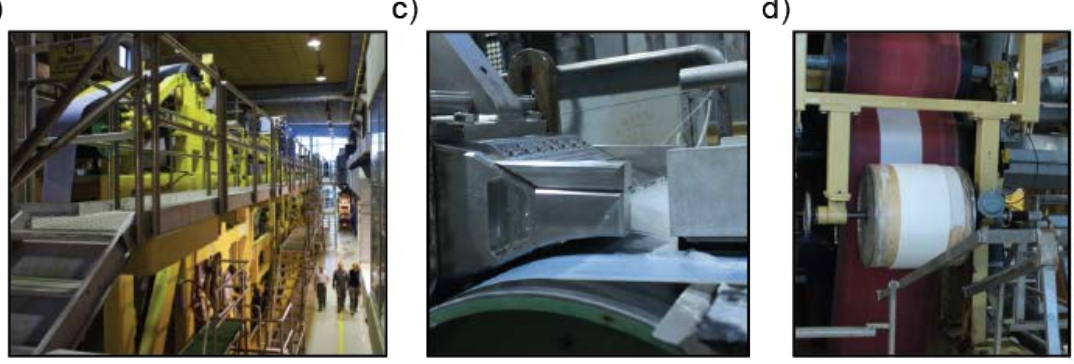

Figure 1. (a) Schematic view of the pilot paper machine. (b) Photograph of the FEX pilot paper machine. (c) Inlet box supplying the composite pulp. (d) Bobbins collecting the composite paper before drying.

\section{Composite pulp materials}

Panatetra WZ-0501, "tetrapodal ZnO whiskers" ZnO-Ts (single crystal, 99.999\% purity), having an average length of approximately $20 \mu \mathrm{m}$, were purchased from AMTEC KK, Osaka, Japan (figure S1). Previous reports have proven the feasibility of using this form of ZnO in paper composites [33]. We used a mixture of $70 \%$ bleached sulphate softwood pulp and $30 \%$ bleached sulphate hardwood pulp as the cellulose component in the composite. The pulps were co-refined in a 26" double disc mill to $140 \mathrm{kWh} /$ ton using an edgeload of $1.0 \mathrm{Ws} / \mathrm{m}$, resulting in a $28.5^{\circ} \mathrm{SR}$ (Schopper-Riegler) drainage rate. As retention agent we used Perkol 1510 cationic polyacrylamide at the concentration $750 \mathrm{~g} / \mathrm{ton}$, and for the papers containing the sizing agent alkyl ketene dimer wax (AKD) we used $7500 \mathrm{~g} / \mathrm{ton}$. Sizing agents can influence how inks penetrate into the paper. The paper machine was fed with seven different compositions of the composite according to table 1 . First, we prepared a reference paper, A, having no ZnO-Ts and no alkyl ketyl dimer (AKD). The ZnO-Ts content was then increased in steps of $15 \%$ weight percentage (wt\%), first without, and then with AKD for each ZnO-Ts content, to form paper types B-G. In all samples the feed was aimed at a grammage of $60 \mathrm{~g} / \mathrm{m}^{2}$. 


\subsection{Electrode printing}

To probe the photoconductivity of the composite paper and to develop photosensor devices, we printed electrodes using conducting carbon-based ink (7102, supplied by DuPont) (Figure 2(a)). Two-probe interdigitated electrodes and four-probe square configured electrodes were designed and transferred to a screen-printing mask (figure 2(b) and figure S2 in Supporting Information [SI], respectively). Two-probe interdigitated electrode devices (designed for $0.25 \mathrm{~mm}$ electrode width and distance and a total foot print of around $0.5 \mathrm{~cm}^{2}$ ) were used to measure the photosensing properties of the composite paper while the four-probe electrode devices were used to measure the intrinsic properties of the material, such as the sheet conductance and the bulk conductivity. For the latter, square arrangement of probes was preferred to avoid shadowing of the photoconductor by the probes or electrodes. The screen-printing mask was a $20 \mu \mathrm{m}$ capillary film trademarked Fotecoat 1177 on a polyester web with 120 threads/cm. After printing, the paper with the ink was dried at $100^{\circ} \mathrm{C}$ in an oven for 3 minutes.

\subsection{Photoconductivity measurements}

Photoconductive measurements were performed using a LCS-100 Solar Simulator from Oriel Instruments as the light source. The system uses a $100 \mathrm{~W}$ Xe lamp and an AM1.5G filter. The irradiance was 1 Sun $\left(1000 \mathrm{~W} / \mathrm{m}^{2}\right)$ unless stated otherwise. The calibration of the light irradiance was done by using a calibrated reference cell and meter (91150-KG5 from Oriel Instruments). The voltage applied throughout the photosensing characterization of the composite paper was $1 \mathrm{~V}$ unless stated otherwise. The current was measured and recorded in one-second intervals with a Keithley 2420 sourcemeter. A custom-designed LabVIEW program was used to control the equipment and gather the data on a personal computer. The sheet resistance of the paper material was obtained by illuminating the four-probe structure through a circular shadow mask, where the periphery coincides with the electrodes, and then calculated by using the square fourprobe points configuration formula [34].

\section{Results and discussion}

\subsection{Material Fabrication and Structural characterization}

ZnO-Ts cellulose composite papers were successfully manufactured on the FEX pilot paper machine, with dry contents of ZnO-Ts up to $45 \mathrm{wt} \%$. The grammage and composition of the composite papers are shown in table 1 . In all papers, the grammage is near the target value of $60 \mathrm{~g} / \mathrm{m}^{2}$, with slightly lower values for sample $\mathrm{F}$ and $\mathrm{G}$ that had the highest loading of $\mathrm{ZnO}-\mathrm{Ts}$. The content of inorganic content in a paper can be provided by the ash content, as the cellulose component leaves very little ash upon combustion. Indeed, the ash content in the reference paper A was only $0.3 \%$. The ash contents in the ZnO-Ts loaded samples (B-G), are consistent with the targeted compositions. In the papers with the highest loading of ZnO-Ts, one can see a slightly lower ash content than the targeted ZnO-Ts content. This together with a slightly lower grammage than the target value indicates lower retention in papers $F$ and $G$. Samples $D$ and $E$, on the other hand, have grammage and ash content consistent with an acceptable retention in the paper making process. Paper $\mathrm{E}$ was therefore selected for further characterization. 
Table 1. Targeted ZnO-content and measured grammage and ash content of the produced composite papers.

\begin{tabular}{ccccc}
\hline Sample & $\begin{array}{c}\text { Targeted ZnO } \\
\text { content (\%) }\end{array}$ & $\begin{array}{c}\text { AKD addition } \\
\text { (yes / no) }\end{array}$ & $\begin{array}{c}\text { Measured } \\
\text { Grammage }^{\mathbf{a}} \mathbf{( g / \mathbf { m } ^ { 2 } )}\end{array}$ & $\begin{array}{c}\text { Ash content }^{\mathbf{b}} \\
\mathbf{5 5 0} \mathbf{C} \mathbf{~ ( \% )}\end{array}$ \\
\hline A & 0 & no & 60,8 & 0,3 \\
B & 15 & no & 62,6 & 15,9 \\
C & 15 & yes & 63,2 & 15,7 \\
D & 30 & no & 62,0 & 30,8 \\
E & 30 & yes & 60,5 & 31,1 \\
F & 45 & no & 56,1 & 44,5 \\
G & 45 & yes & 57,3 & 44,1 \\
\hline
\end{tabular}

a Determined according to ISO 536:2012.

${ }^{b}$ Determined according to ISO 1762 , where ashing is performed by vaporizing/combusting organic compounds at $550{ }^{\circ} \mathrm{C}$. ZnO is stable at this temperature.

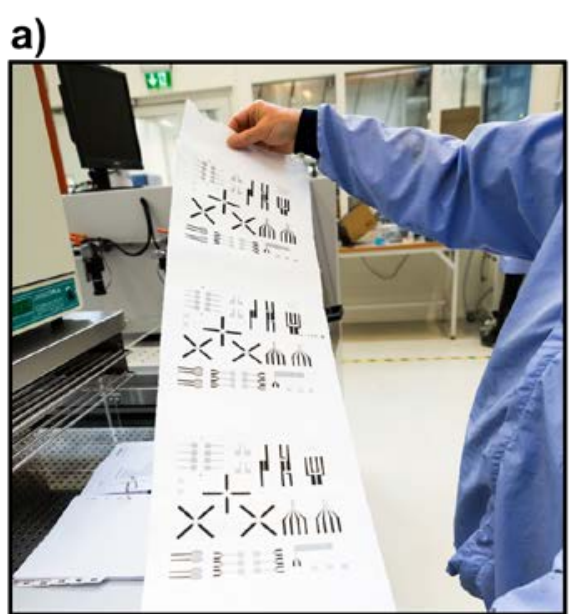

b)

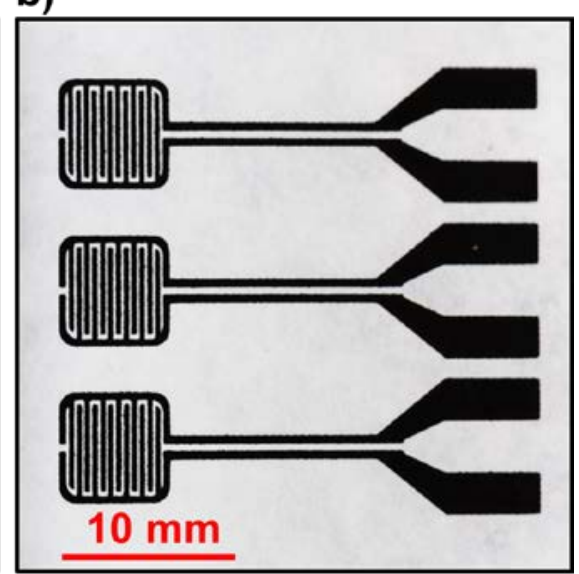

c)

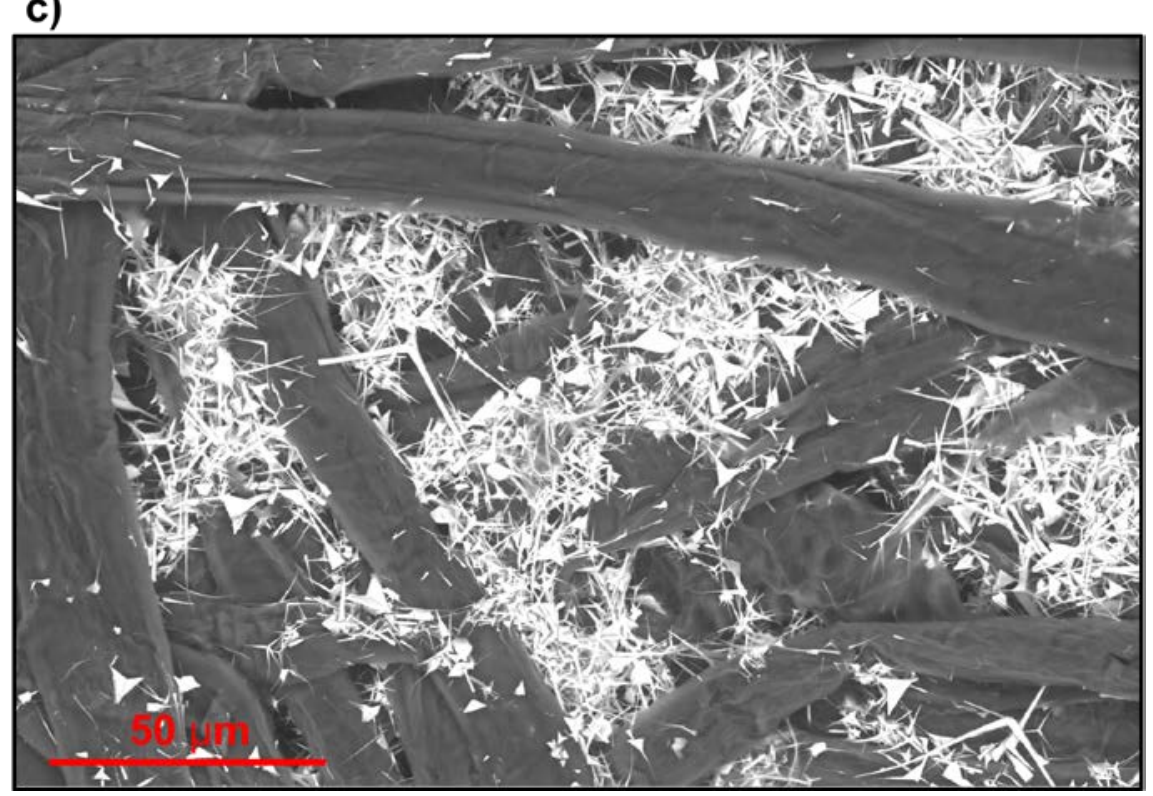

Figure 2. (a) Photograph of ZnO-composite paper with printed devices. (b) Two-probe ZnOcomposite devices, as used for photosensing. (c) SEM image of the ZnO-composite paper (paper E). 
The papers functioned well as substrates for screen-printing of carbon-based electrodes, as seen in figure 2(a) and 2(b). In order to study the arrangement of the ZnO-Ts in the cellulose matrix we took SEM images of the ZnO-composite paper (figure 2(c)). It appears that the ZnOTs successfully fill voids between the fibers in the paper. One concern about the manufacturing of the composite paper was release of ZnO-Ts particles from the paper during manufacturing. However, very little visible traces of particle release from the paper were observed in the wet stage of the machine and also not at the drying section. This is in agreement with the high retention indicated by the measured grammage and ash content. This may be the result of large ZnO-Ts that become entangled with the wood fiber network composed of both long and short fibers. However, we noticed that some traces of powder can detach from the paper if we shake, bend or crease the paper. This may be minimized in the future through further optimization of the paper production protocol, where we speculate that the addition of finely ground parts of fibers called fines may help to further hold the ZnO-Ts together and bind them to the paper [35].

\subsection{Photosensing Characterization}

We evaluate the ZnO-based paper as photosensor using the two-probe interdigitated electrode configuration shown in figure 2(b). The red curve in figure 3(a) shows the measured current of the device at a bias of $1 \mathrm{~V}$, first without light and followed by illumination at 1 Sun of irradiance. As expected, the averaged dark current exhibits a very low value of $8.5 \cdot 10^{-4} \mu \mathrm{A}$ while illumination results in a dramatic increase in the measured current. This photoresponse behaviour can be explained by the well-known mechanism of oxygen adsorption-desorption, as discussed above $[7,26,28]$. The results corroborate that the density and connectivity of ZnO-Ts in the material is high enough and favourable, respectively, to allow for long-range conduction through the material. Interestingly, the current immediately reaches high values upon illumination, followed by a slow decrease to a stable signal of $12 \mu \mathrm{A}$ after around 20 minutes. The resulting photo-dark current ratio is well above 14000. The photoresponse behaviour makes sensors based on our composite paper particularly suitable for applications where light doses need to be measured over longer times and on large surfaces, such as food preservation, environmental UV-dosage monitoring, or crop growth control. However, the strong immediate photoresponse will also enable measurements of short light exposures. We note that the stabilization curve (red curve of figure 3(a)) was obtained by first applying the voltage, followed by illumination. If we instead pre-illuminate the device for a given time before applying the voltage (green and blue curves, $10 \mathrm{~min}$ and $45 \mathrm{~min}$ respectively), the signal becomes stable after correspondingly shorter times (effectively only measuring the last part of the full stabilization curve, see figure 3(a)). This indicates that the stabilization effect is not a bias-stress effect in part caused by the applied voltage, but rather related to the illumination. Our results are in agreement with previous studies that report that the evolution of the photocurrent for lowdimensional $\mathrm{ZnO}$ structures includes a fast increase upon illumination, followed by a slow decay that asymptotically stabilizes at a current level given by the specific illumination intensity [36,37]. In turn, the photocurrent decay part has been found related to two processes with distinctly different time constants [37]. The relatively faster process (time constant $\sim 50-300 \mathrm{~s}$ ) could be attributed to trapping of photoelectrons by $\mathrm{O}_{2}$ molecules that are re-adsorbed on the surface, while the relatively slower process (time constant $>500 \mathrm{~s}$ ) could be attributed to recombination at native defects $[38,39]$. The details of these processes have also been found dependent on the substrate onto which the $\mathrm{ZnO}$ structure are carried as well as on the presence of $\mathrm{H}_{2} \mathrm{O}$ and $\mathrm{O}_{2}$ in the surrounding environment [39]. 
Figure 3(b) shows the current response for three consecutive illumination on/off cycles after the stabilization period. The results demonstrate a very repeatable behaviour, with minimal dark current and a stable photocurrent of $12 \mu \mathrm{A}$ (red curve). The rise time is defined as the time required for the current to increase from $10 \%$ to $90 \%$ of the saturated value, and the decay time as the time required for the current to decrease from $90 \%$ to $10 \%$ [26]. The device shows an average rise time and decay time of $15 \mathrm{~s}$ and $6 \mathrm{~s}$, respectively (figure 3(c)). The values obtained are highly competitive when compared with other response times reported in the literature on small-scale nanostructured $\mathrm{ZnO}$ photosensors. For instance, photodetectors based on $\mathrm{ZnO}$ single nanowires showed a rise and decay time of $53 \mathrm{~s}(80 \%)$ and $300 \mathrm{~s}(1.5 \%)$, respectively [40]. ZnO nanorods prepared by sol-gel showed response times of $3.7 \mathrm{~s}$ and $63.7 \mathrm{~s}$ respectively [41]. ZnO devices prepared by chemical bath deposition showed response times of $9 \mathrm{~s}$ and $21 \mathrm{~s}$, respectively [26]. On flexible ZnO-nanoparticle-assembled cloth the reported response times were $3 \mathrm{~s}$ and $2 \mathrm{~s}$, respectively [28]. Interestingly, the decay time for our device is lower than the rise time. This finding is opposite to the observations of several previous reports for $\mathrm{ZnO}$-based photosensors, in which the decay time was found longer than the rise time, as attributed to a socalled persistent photoconductivity due to trap states within the ZnO bandgap $[26,42,43]$. The faster decay time than rise time for our ZnO-Ts paper indicates that the adsorption of oxygen molecules is faster than the desorption process upon illumination. This may be due to defects on the material that causes a slow movement of the photo-generated holes from the bulk to the surface of the material. It should also be noted that the photoresponse was found linear with applied bias potential, indicating ohmic contacts in our device, and that the electrons generated by photon excitation are easily transported into the printed carbon electrodes (see figure S3) [44]. The photoresponse was also measured on a blank sample without ZnO-Ts (Paper A). Upon illumination, the device made from this blank paper shows no observable response beyond the noise level (blue curve of figure 3(b)).

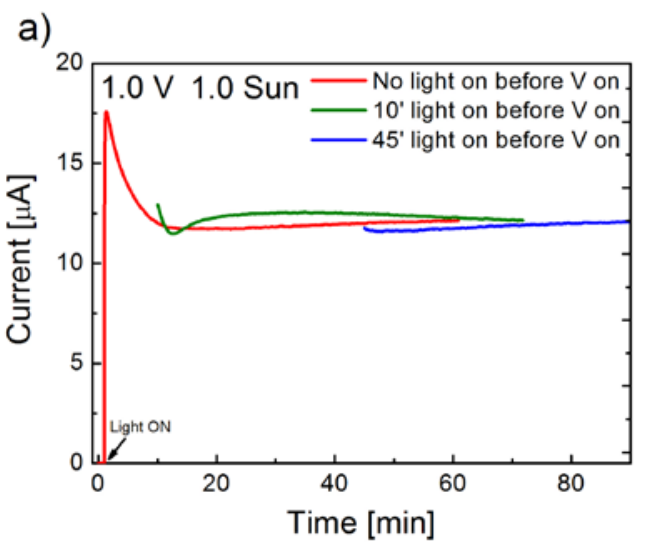



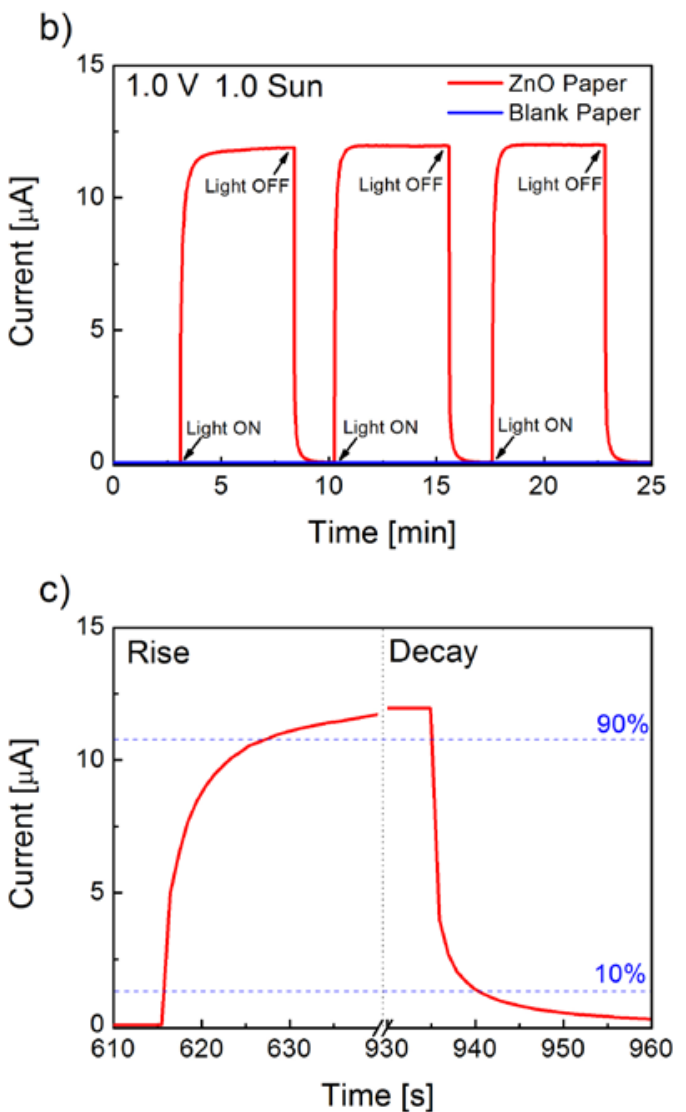

Figure 3. (a) Stabilization curve of the ZnO-Ts paper at a bias of $1 \mathrm{~V}$ and under an irradiance of 1 Sun (red line). The green and blue lines were measured by pre-illumination of the device for 10 min and 45 min, respectively, before applying the voltage. (b) Photoresponse of the device at a bias of $1 \mathrm{~V}$ and for alternating dark conditions and irradiance at 1 Sun (red line). The blue curve shows the photoresponse for a blank sample, respectively. (c) Enlarged view of figure3(b) showing the rise and decay times of one of the cycles (second cycle) of the device.

Next, we characterize the sensitivity of the devices by investigating the photoresponse at different light intensities. These experiments were performed by changing the illumination irradiance while keeping a fixed bias voltage of $1 \mathrm{~V}$ (figure 4(a)). Figure 4(b) shows the averaged stable current measured at different irradiances. The device shows a strong photoresponse for light irradiances spanning at least two orders of magnitude (0.01-1 Sun). The dependence of the current on light irradiance can be explained by a model accounting for a total device resistance $(R)$ that is the sum of a constant resistance originating from leads and contacts $\left(R_{\mathrm{c}}\right)$, and a lightdependent resistance through the paper $\left(R_{\mathrm{p}}\right)$. The latter is set inversely proportional to the irradiance $(E)$, as motivated by four-probe measurements of the ZnO-paper material. The fourprobe measurements eliminate effects from contacts and demonstrate that the photocurrent (and hence, the conductivity) of the paper varies linearly with illumination irradiance (inset in figure $4(b)$ ). The stable current $(I)$ of the interdigitated photosensor device should then depend on light irradiance $(E)$ as

$$
I=\frac{V}{S / E+R}
$$

where $S$ is a sensitivity factor determined by both device geometry and the inherent sensitivity of the paper's conductivity to illumination. The experimental data can be excellently fitted to this 
expression (red curve in figure 4(b)). The fit enables extraction of the total contact and lead resistance of the device $\left(R_{\mathrm{c}}\right)$ as $74 \mathrm{k} \Omega$, while the total resistance within the paper $\left(R_{\mathrm{p}}=S / E\right)$ is found to decrease inversely with illumination as $11 \mathrm{k} \Omega \times$ Sun. This reflects the significant resistance of the printed carbon in electrodes and leads, a material choice motivated by the low cost of carbon and its ohmic contact to ZnO. The current varies strongly and approximately linearly with irradiance at low light intensities, where $R_{\mathrm{p}}$ dominates the device resistance. This could be beneficial for low light intensity applications. We also note that both $S$ and $R_{\mathrm{c}}$ are dependent on device geometry, enabling the response curve to be modified and optimized for different applications and illumination conditions.

From the four-probe measurements, we extract the sheet resistance of the $\mathrm{ZnO}$-Ts paper as $6.9 \cdot 10^{7} \Omega /$ sq under a bias voltage of $1 \mathrm{~V}$ and at 1 Sun of irradiance. For our roughly $100 \mu \mathrm{m}$ thick paper, and approximating the light intensity as constant through the paper, this gives a bulk conductivity of $1.5 \cdot 10^{-4} \mathrm{~S} / \mathrm{m}$ under a bias of $1 \mathrm{~V}$ and at $1 \mathrm{Sun}$.

a)

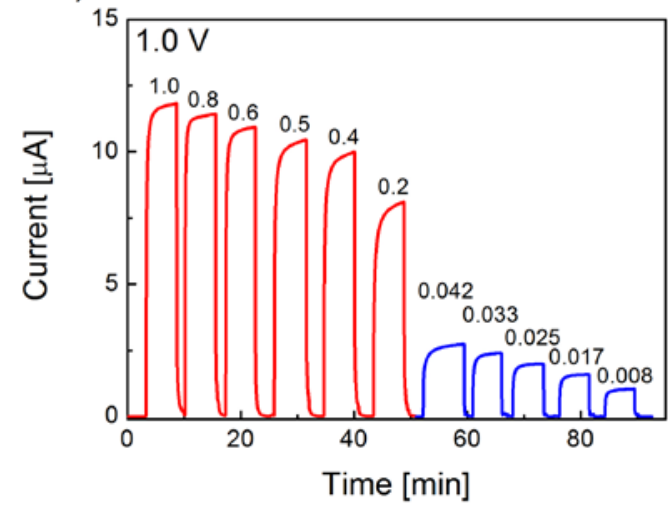

b)

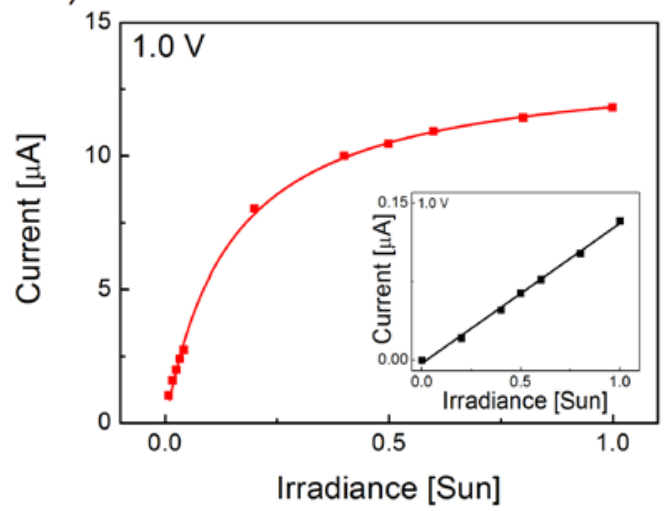

Figure 4. (a) Current at different irradiances over time of the device under a bias of $1 \mathrm{~V}$. The values stated for illumination cycle correspond to the irradiance in Sun. The data points of Figure $4 b$ are extracted from the average of the current at the different intensities of light. (b) Current versus irradiance of the device under a bias of $1 \mathrm{~V}$. The fit of the photoexcited current is shown (red line). Inset: Current versus irradiance of a four-probe device under a bias of $1 \mathrm{~V}$. The black line is a linear fit of the photoexcited current.

Finally, we demonstrate our device at a large scale, using an over $1 \mathrm{~m}$ long ZnO-composite paper with arrays of different printed carbon electrodes (figure 5(a)). To simulate relevant conditions for real applications, we measure the response outdoor on a partly cloudy day. Using the same two-probe interdigitated device and experimental setup as for the examples above, outdoor ambient light conditions gave a stable photocurrent of $9.1 \mu \mathrm{A}$ under a bias of $1 \mathrm{~V}$ (figure 5(b)). This is around 25\% lower than that measured for simulated solar illumination at 1 Sun, in agreement with the non-ideal Swedish summer weather. When the device is covered, the current decreased to $0.002 \mu \mathrm{A}$ (figure 5(c)). It should be noted here that the photocurrent is dependent on device geometry and larger photocurrents could easily be obtained by modifying the device geometry (figure S3), allowing for optimization of the device for a wide range of applications. 

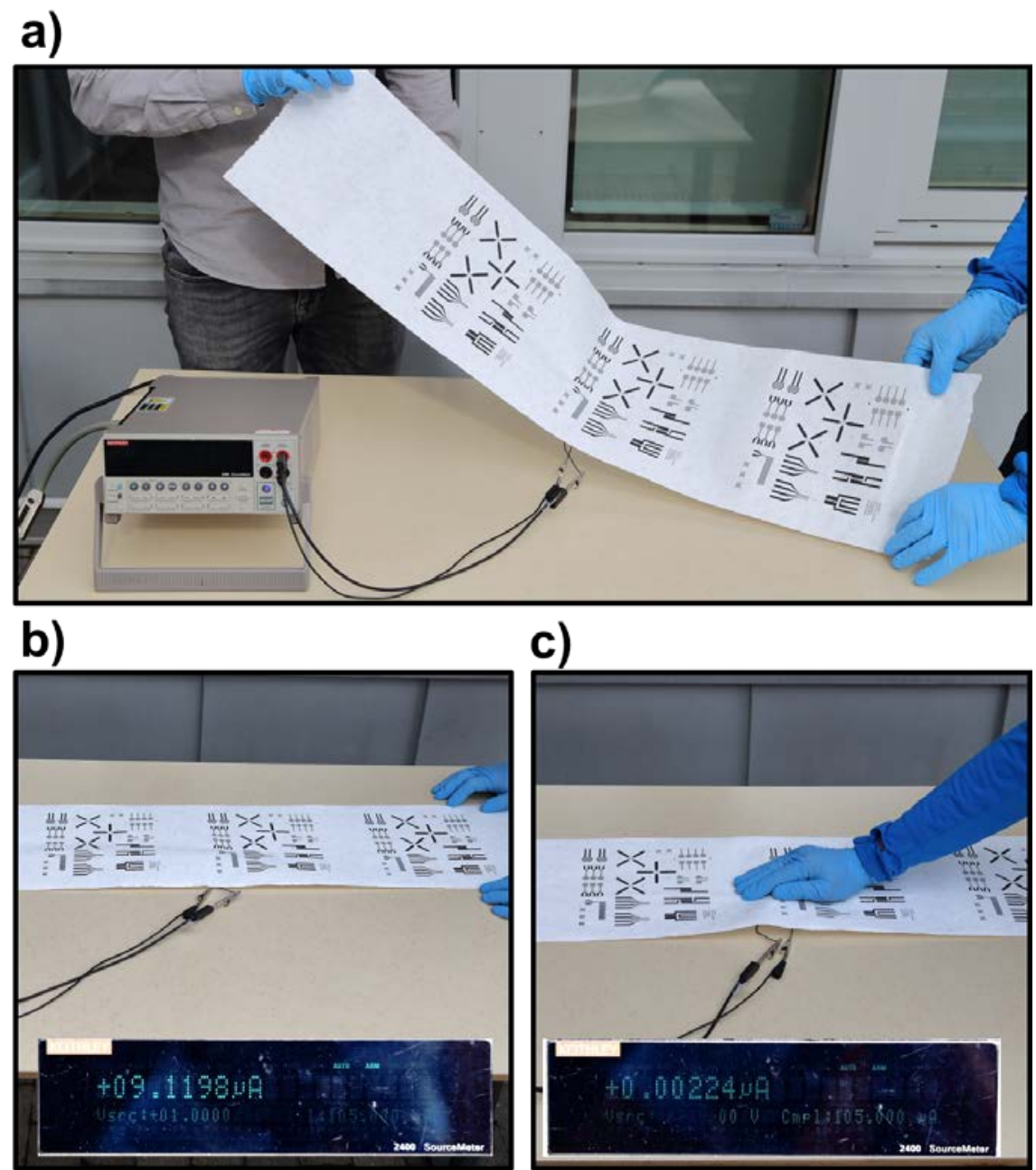

c)

Figure 5. (a) Outdoor setup using a large scale ZnO-composite paper sample. (b) Photocurrent of the device under ambient illumination. (c) Photocurrent of the device when light is blocked.

\section{Conclusions}

To our knowledge, this work presents the first production of an optoelectronic paper material with a pilot paper machine. More specifically, we successfully made functional ZnO-cellulose composite papers with a high content of ZnO-Ts (up to $45 \mathrm{wt} \%$ ). The production rate of $100 \mathrm{~m} / \mathrm{min}$ corresponds to the lowest speed of the pilot paper machine, indicating additional potential for up-scaling. Furthermore, the material flow and processes in the pilot paper machine are very similar to that of a full-scale paper machine, and the raw materials are both available in bulk amounts and cheap compared to typical active optoelectronic materials. Hence, the manufacturing experiment shows that scaling also to full-scale paper machines is technically and economically viable.

The optoelectronic functionality of the composite paper was demonstrated by application as a photosensor. The measured photoresponse of a two-probe interdigitated carbon electrode printed on the paper (with only $0.5 \mathrm{~cm}^{2}$ footprint) showed strong and repeatable photocurrent signals upon illumination with simulated sunlight, with values of $12 \mu \mathrm{A}$ at $1 \mathrm{~V}$. The device showed fast response times, sufficient for monitoring of, for example, the sunlight exposure during a day, and to monitor illumination of light sensitive applications, such as food preservation, human health, crop control, or water purification. 
Scalability to paper-machine manufacturing of papers with optoelectronic functionality opens up for a new concept in large area smart materials, in which composite papers provide functionality within its bulk, and not just act as passive printing substrates. Together with other printed materials and conventional silicon-based devices, this type of smart composite papers can be useful for a large variety of low-cost and large-area devices.

\section{Acknowledgements}

Anders Mähler, Lennart Hermansson, Thomas Sohlberg, Peter Widlund, Daniel Hissing, Görgen Lundström, of Innventia $A B$, are acknowledged for planning and performing the paper production, Joanna Hornatowska for microscope imaging, and Thor Balkhed for photographs of devices. The authors acknowledge financial support from the Knut and Alice Wallenberg foundation in the Power Papers Project, Research Institutes of Sweden (RISE), the WennerGren Foundations, the Swedish Research Council, the Swedish Foundation for Strategic Research, the ÅForsk Foundation and the Swedish Government Strategic Research Area in Materials Science on Functional Materials at Linköping University (Faculty Grant SFO-Mat-LiU No 2009 00971).

\section{References}

[1] Tobjörk D and Österbacka R 2011 Paper Electronics Adv. Mater. 23 1935-61

[2] Statista 2013 Paper industry - Statista Dossier (http://www.statista.com/topics/1701/paperindustry/) Retrieved 14th July 2016

[3] Khan S, Lorenzelli L and Dahiya R S 2015 Technologies for Printing Sensors and Electronics Over Large Flexible Substrates: A Review IEEE Sens. J. 15 3164-85

[4] Perelaer J, Smith P J, Mager D, Soltman D, Volkman S K, Subramanian V, Korvink J G and Schubert U S 2010 Printed electronics: the challenges involved in printing devices, interconnects, and contacts based on inorganic materials J. Mater. Chem. 20 8446-53

[5] Griesmer J J 1978 Accurate and Meaningful Conductivity Measurements Tappi 61 97-9

[6] Friedrich R E and Chiu T T 1970 Comparison of AC and DC Methods of Measuring Conductivities in Electrophotographic Papers Tappi 53 282-4

[7] Soci C, Zhang A, Xiang B, Dayeh S A, Aplin D P R, Park J, Bao X Y, Lo Y H and Wang D 2007 ZnO Nanowire UV Photodetectors with High Internal Gain Nano Lett. 7 1003-9

[8] Melnick D A 1957 Zinc Oxide Photoconduction, an Oxygen Adsorption Process J. Chem. Phys 26 1136-46

[9] Collins R J and Thomas D G 1958 Photoconduction and Surface Effects with Zinc Oxide Crystals Phys. Rev. 112 388-95

[10] Willander M 2014 Zinc Oxide Nanostructures: Advances and Applications Singapore Pan Stanford

[11] Schmidt-Mende L and MacManus-Driscoll J L 2007 ZnO - nanostructures, defects, and devices Mater. Today 10 40-8

[12] Kołodziejczak-Radzimska A and Jesionowski T 2014 Zinc Oxide-From Synthesis to Application: A Review Materials 7 2833-81

[13] Özgür Ü, Hofstetter D and Morkoc H 2010 ZnO Devices and Applications: A Review of Current Status and Future Prospects Proc. IEEE 98 1255-68

[14] Özgür Ü, Alivov Y I, Liu C, Teke A, Reshchikov M A, Dogan S, Avrutin V, Cho S J and Morkoc H 2005 A comprehensive review of ZnO materials and devices J. Appl. Phys. 98041301

[15] Wang Z L 2004 Zinc oxide nanostructures: growth, properties and applications J. Phys. Condens. Matter 16 R829-R58

[16] Office U G P 2015 CFR - Code of Federal Regulations Title 21 (https://www.accessdata.fda.gov/scripts/cdrh/cfdocs/cfcfr/CFRSearch.cfm?CFRPart=182)

Retrieved 14th July 2016 
[17] Sivamani R K, Crane L A and Dellavalle R P 2009 The Benefits and Risks of Ultraviolet Tanning and Its Alternatives: The Role of Prudent Sun Exposure Dermatol. Clin. 27 149-54 [18] Cooper K D, Oberhelman L, Hamilton T A, Baadsgaard O, Terhune M, Levee G, Anderson $\mathrm{T}$ and Koren $\mathrm{H} 1992 \mathrm{Uv}$ exposure reduces immunization rates and promotes tolerance to epicutaneous antigens in humans - relationship to dose, cd1a-dr+epidermal macrophage induction, and langerhans cell depletion Proc. Natl. Acad. Sci. 89 8497-501

[19] Hader D P, Helbling E W, Williamson C E and Worrest R C 2011 Effects of UV radiation on aquatic ecosystems and interactions with climate change Photochem. Photobiol. Sci. 10 242-60 [20] Teramura A H, Ziska L H and Sztein A E 1991 Changes in growth and photosynthetic capacity of rice with increased UV-B radiation Physiol. Plant. 83 373-80

[21] Guerrero-Beltrán J A and Barbosa-Cánovas G V 2004 Advantages and Limitations on Processing Foods by UV Light Food Sci. Tech. Int. 10 137-47

[22] Lim W and Harrison M A 2016 Effectiveness of UV light as a means to reduce Salmonella contamination on tomatoes and food contact surfaces Food Control 66 166-73

[23] Johnson K M, Kumar M R A, Ponmurugan P and Gananamangai B M 2010 Ultraviolet radiation and its germicidal effect in drinking water purification J. Phytol. 2 12-9

[24] Jun J H, Seong H, Cho K, Moon B-M and Kim S 2009 Ultraviolet photodetectors based on ZnO nanoparticles Ceram. Int. 35 2797-801

[25] Liu X, Gu L, Zhang Q, Wu J, Long Y and Fan Z 2014 All-printable band-edge modulated ZnO nanowire photodetectors with ultra-high detectivity Nat. Commun. 54007

[26] Shaikh S K, Inamdar S I, Ganbavle V V and Rajpure K Y 2016 Chemical bath deposited ZnO thin film based UV photoconductive detector J. Alloys Compd. 664 242-9

[27] ul Hasan K, Nur O and Willander M 2012 Screen printed ZnO ultraviolet photoconductive sensor on pencil drawn circuitry over paper Appl. Phys. Lett. 100211104

[28] Liu B et al. 2012 ZnO-nanoparticle-assembled cloth for flexible photodetectors and recyclable photocatalysts J. Mater. Chem. 22 9379-84

[29] Gimenez A J, Yáñez-Limon J M and Seminario J M 2013 ZnO-Cellulose Composite for UV Sensing IEEE Sens. J. 13 1301-6

[30] Gimenez A, Luna-Bárcenas G, Sanchez I and Yánez-Limón J 2015 Paper-Based ZnO Oxygen Sensor IEEE Sens. J. 15 1246-51

[31] Roding S and Norman B 1986 FEX, the new STFI experimental paper machine Tappi 69 94-7

[32] Nordström B 1995 Effects of headbox design and dewatering conditions on twin-wire forming of TMP (PhD Thesis) Stockholm KTH - Royal Institute of Technology, Department of pulp and paper chemistry and technology

[33] Koga H, Kitaoka T and Wariishi H 2008 In situ synthesis of Cu nanocatalysts on ZnO whiskers embedded in a microstructured paper composite for autothermal hydrogen production Chem. Commun. 5616-8

[34] Miccoli I, Edler F, Pfnür H and Tegenkamp C 2015 The 100th anniversary of the four-point probe technique: the role of probe geometries in isotropic and anisotropic systems J. Phys.

Condens. Matter 27223201

[35] Taipale T, Österberg M, Nykänen A, Ruokolainen J and Laine J 2010 Effect of microfibrillated cellulose and fines on the drainage of kraft pulp suspension and paper strength Cellulose 17 1005-20

[36] Ahn S-E et al. 2007 Origin of the slow photoresponse in an individual sol-gel synthesized ZnO nanowire Appl. Phys. Lett. 90 153106-3

[37] Bera A and Basak D 2008 Carrier relaxation through two-electron process during photoconduction in highly UV sensitive quasi-one-dimensional ZnO nanowires Appl. Phys. Lett. 93 053102-3

[38] Bera A and Basak D 2009 Role of defects in the anomalous photoconductivity in ZnO nanowires Appl. Phys. Lett. 94 163119-3

[39] Bera A and Basak D 2011 Pd-nanoparticle-decorated ZnO nanowires: ultraviolet photosensitivity and photoluminescence properties Nanotech. 22 265501-7 
[40] Li P-J, Liao Z-M, Zhang X-Z, Zhang X-J, Zhu H-C, Gao J-Y, Laurent K, Leprince-Wang Y, Wang N and Yu D-P 2009 Electrical and Photoresponse Properties of an Intramolecular p-n Homojunction in Single Phosphorus-Doped ZnO Nanowires Nano Lett. 9 2513-8

[41] Ahn S E, Lee J S, Kim H, Kim S, Kang B H, Kim K H and Kim G T 2004 Photoresponse of sol-gel-synthesized ZnO nanorods Appl. Phys. Lett. 84 5022-4

[42] Hullavarad S, Hullavarad N, Look D and Claflin B 2009 Persistent Photoconductivity Studies in Nanostructured ZnO UV Sensors Nanoscale Res. Lett. 4 1421-7

[43] Cammi D and Ronning C 2014 Persistent Photoconductivity in ZnO Nanowires in Different Atmospheres Adv. Cond. Matter. Phys. 2014184120

[44] Yang Z, Wang M, Song X, Yan G, Ding Y and Bai J 2014 High-performance ZnO/Ag Nanowire/ZnO composite film UV photodetectors with large area and low operating voltage $J$. Mater. Chem. C 2 4312-9 